\title{
A New Approach Based on Iterative Method for the Characterization of a Micro-Strip Line with Thick Copper Conductor
}

\author{
Rafika Mejri, Taoufik Aguili \\ Communication System Laboratory Sys'Com, National Engineering School of Tunis, University Tunis El Manar, \\ Tunis, Tunisia \\ Email: mejrirafika@yahoo.fr
}

Received 20 April 2016; accepted 27 May 2016; published 30 May 2016

Copyright (C) 2016 by authors and Scientific Research Publishing Inc.

This work is licensed under the Creative Commons Attribution International License (CC BY). http://creativecommons.org/licenses/by/4.0/

c) (i) Open Access

\begin{abstract}
In this work, we applied two electromagnetic models for the characterization of a planar structure including a flat, thick copper conductor. Indeed the first model is consisted by modeling two metal ribbons without bulkiness, placed one above the other at a distance of $h_{2}$ equal to the thickness of the thick conductor. This approach has been implemented and tested by the iterative method. The results of simulations have been compared with those calculated by the Ansoft HFSS software, and they are in good concordance, validating the method of analysis used. The second model is based on the calculation of the effective permittivity of the medium containing the thick conductor. This medium consists of a metallic region of complex relative permittivity $\tilde{\varepsilon}^{\prime \prime}$, the rest of this medium is filled with air $\varepsilon_{r 2}=1$. The effective permittivity $\varepsilon_{\text {eff }}$ calculated from these two relative permittivity $\varepsilon_{\mathrm{r} 2}$ and $\tilde{\varepsilon}^{\prime \prime}$. Comparing the simulation results of this new formulation of the iterative method with those calculated by the software Ansoft HFSS shows that they are in good matching which validates the second model.
\end{abstract}

\section{Keywords}

Thick Conductor, Iterative Method, Micro-Strip Line, Microwaves, Skin Effect Phenomenon, Effective Permittivity

\section{Introduction}

In Radio frequency, most devices are made in micro strip technology [1] [2]. This technology became the best known and most used, this is due to its flat nature, ease of manufacturing, low cost, easy integration with circuits 
in the solid state, good heat dissipation structure that is used as good mechanical support etc. In general and in various research works concerning modeling and study of these structures, most researchers assumed that they possessed metallic conductors without thickness [3] [4]. This simplifying assumption decreases the accuracy of the results of analytical methods used for the characterization of these structures. Several methods were used to characterize the influence of the thickness of the conductor used, such as mode-matching method [5], method of lines [6], spectral domain method [7] and conformal mapping method [8]. For the study of structures with flat and thick conductors such as micro strip line in this document, we have taken the first model proposed in [6]. A new formulation of the iterative method FWCIP (Fast Wave Concept Iterative Process) was made to extend the study of planar structures with thick flat conductor. This method is based on the concept of wave [9]. It is developed for the simple planar layer modeling of structures [10] or multi-layer [11], and even arbitrarily complex shape. This is an easy method to implement due to the absence of test functions. It is always convergent and has considerable execution speed due to the FMT (Fast Modal Transform).

\section{Iterative Method F.W.C.I.P}

This method is well suited to the calculation of planar structures. In fact, TE and TM modes are used in the iterative method as digital basis of spectral domain in which the FFT. Subsequently, the concept of fast wave is introduced to reflect the boundary conditions and continuity of relationships in different parts of the interface $\Omega$ in terms of waves. The method involves determining an effective relationship to link the incident and reflected waves in different dielectric layers expressing thoughts in modal domain and the boundary conditions and continuity, expressed in terms of waves in spatial domain. The iterative process is then used to move from one field to another using the FMT thus accelerating the iterative process and then the convergence of the method. The use of the FMT requires the pixel description of the different regions of the dielectric interfaces [12]. Thus the electromagnetic behavior of a planar structure will be described by writing the boundary conditions and continuity of the tangential fields on each pixel containing the interface circuitry to study. This integral formulation retains the advantages well known iterative methods including ease of implementation and speed of execution.

\section{Formulation of the Iterative Method for the First Model}

\subsection{Skin Effect Phenomenon}

An approach based on skin effect phenomenon for the modeling of a micro-strip line with thick copper conductor [3]-[5]. In fact at high frequency the skin effect phenomenon occurs and the current only flows on the periphery of the conductor. According to the law of Biot and Savart a moving current in a conductor generates a magnetic field $\mathrm{H}$ around it. If an alternating current flows through a conductive wire, fillers vibrate and the field $\mathrm{H}$ varies which creates an induced current loop that opposes to the change of current in the conductor. Therefore, the sum of the alternating current with that of the loop always lowers the center of the driver while the two currents are added to the surface (see Figure 1). The analytical method used is based on the Fast Wave Concept Iterative Process (FWCIP).

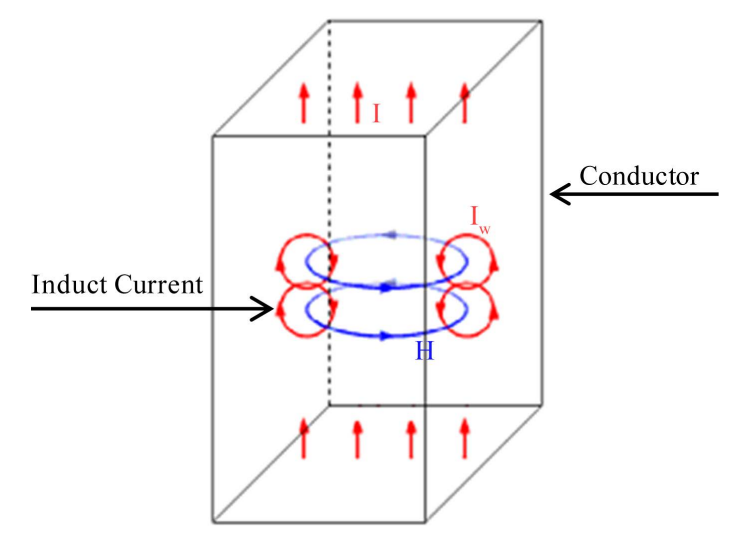

Figure 1. Principle of the skin effect. 


\subsection{Presentation of the Studied Structure}

The studied structure is composed by a dielectric substrate of thickness $H$, on one of its two faces is deposited a metal strip of copper of thickness $T$, the other face is the ground plane of the structure. In fact the current in hyper frequency flows only on the surface of the conductor (see Figure 2). We modeled the thick metal tape by two metal strips without bulkiness placed one above the other at a distance $h_{2}=T=10 \mu \mathrm{m}$. we have $w \gg T$ This allows us to neglect both thick coasts of conductive structure.

Figure 3 presents the variation of skin depth of copper conductor as function of frequency. The value of the skin thickness can be done by applying the following analytical equation:

$$
\delta=\sqrt{\frac{2}{\omega \cdot \mu \cdot \sigma}}=\sqrt{\frac{2 \rho}{\omega \cdot \mu}}=\sqrt{\frac{1}{\pi \cdot \mu \cdot \sigma \cdot f}}
$$

$\delta$ : Skin thickness in meters [m].

$\omega:$ pulsation $[\mathrm{rad} / \mathrm{s}]$.

$f$ : Frequency [Hz].

$\mu$ : magnetic permeability $[\mathrm{H} / \mathrm{m}]$.

$\rho$ : Resistivity $[\Omega \cdot \mathrm{m}]$.

$\sigma$ : Conductivity $[\mathrm{S} / \mathrm{m}]$.

with $\rho=(1 / \sigma)$.

\subsection{Formulation of the Method}

The theoretical formulation for the iterative method is based on determining the relationship between the incident waves " $\vec{A}_{1}, \vec{A}_{21}, \vec{A}_{22}$ and $\vec{A}_{3}$ ” defined in spatial domain and the reflected waves “ $\vec{B}_{1}, \vec{B}_{21}, \vec{B}_{22}$ and $\vec{B}_{3}$ " defined in the spectral domain. The passage of the spatial domain to the spectral domain is using modal Fourier transform (FMT). The passage of the spectral domain to the space domain is using the transform inverse Fourier modal $\left(\mathrm{MTF}^{-1}\right)$. These operations are done with repetitions until the convergence of the method. FMT and $\mathrm{FMT}^{-1}$ are used to speed up the computation time of the method. This evolution is initiated by the waves emitted by the excitation source on either side of the plane $\Omega_{1}$. Figure 4 summarizes the iterative method for planar structures with three layers incorporating different mediums.

$\hat{\Gamma}_{\Omega 1}$ and $\hat{\Gamma}_{\Omega 2}$ : Diffraction operators, giving the incident waves from the reflected waves that diffract at the discontinuities plans $\left(\Omega_{1}\right.$ and $\left.\Omega_{2}\right)$. They are defined in spatial domain and found in these image operators circuits

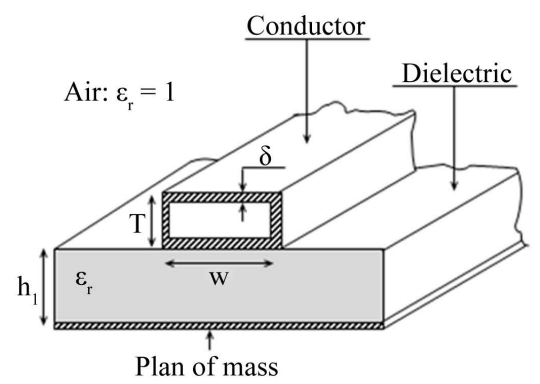

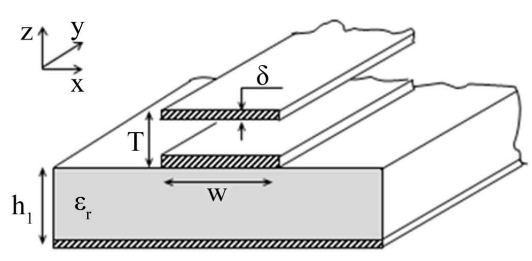

(a)

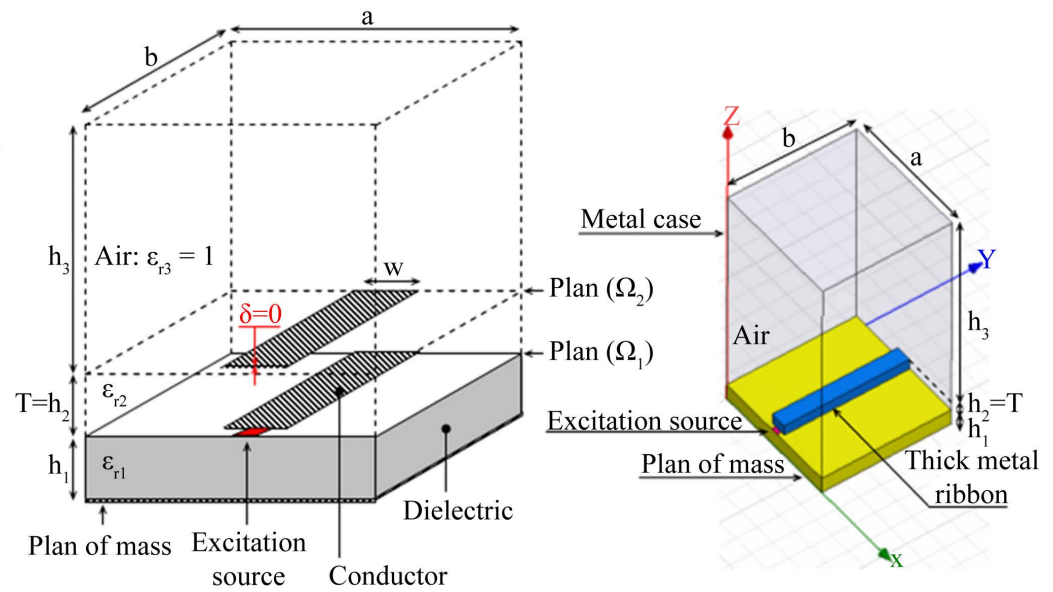

(b)

(c)

Figure 2. Studied structure and offered model based on the skin effect. Parameter of the equivalent circuit structure (first model): $a=18.4 \mathrm{~mm}, b=24 \mathrm{~mm}, c_{1}=0.75 \mathrm{~mm}, d_{1}=0.375 \mathrm{~mm}, w=2.3 \mathrm{~mm}, h_{1}=1.52 \mathrm{~mm}, h_{2}=10 \mu \mathrm{m}, h_{3}=14.99 \mathrm{~mm}, \varepsilon_{r 1}$ $=4.32, \varepsilon_{r 2}=1, \varepsilon_{r 3}=1, \sigma=59.6 \times 10^{6} \mathrm{~S} / \mathrm{m}$. 


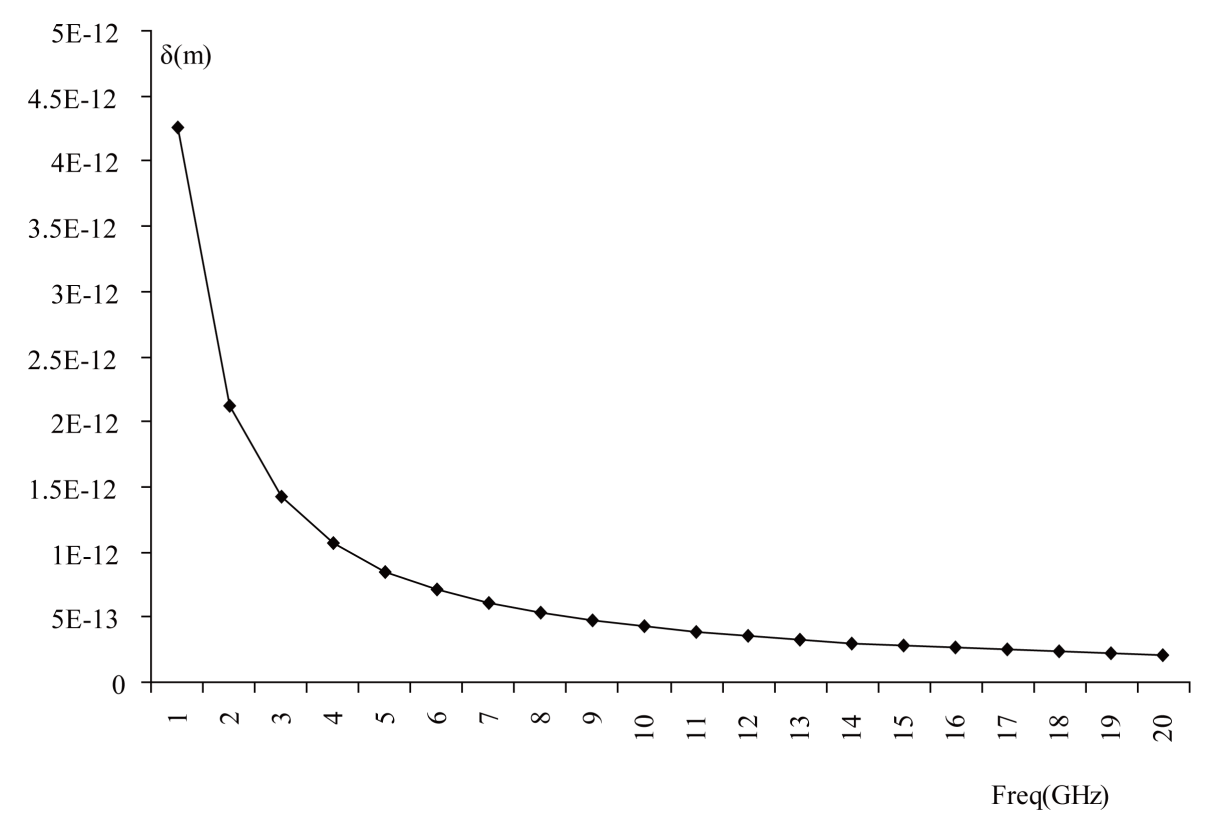

Figure 3. Variation of skin thickness (according in terms as a function) to the frequency.

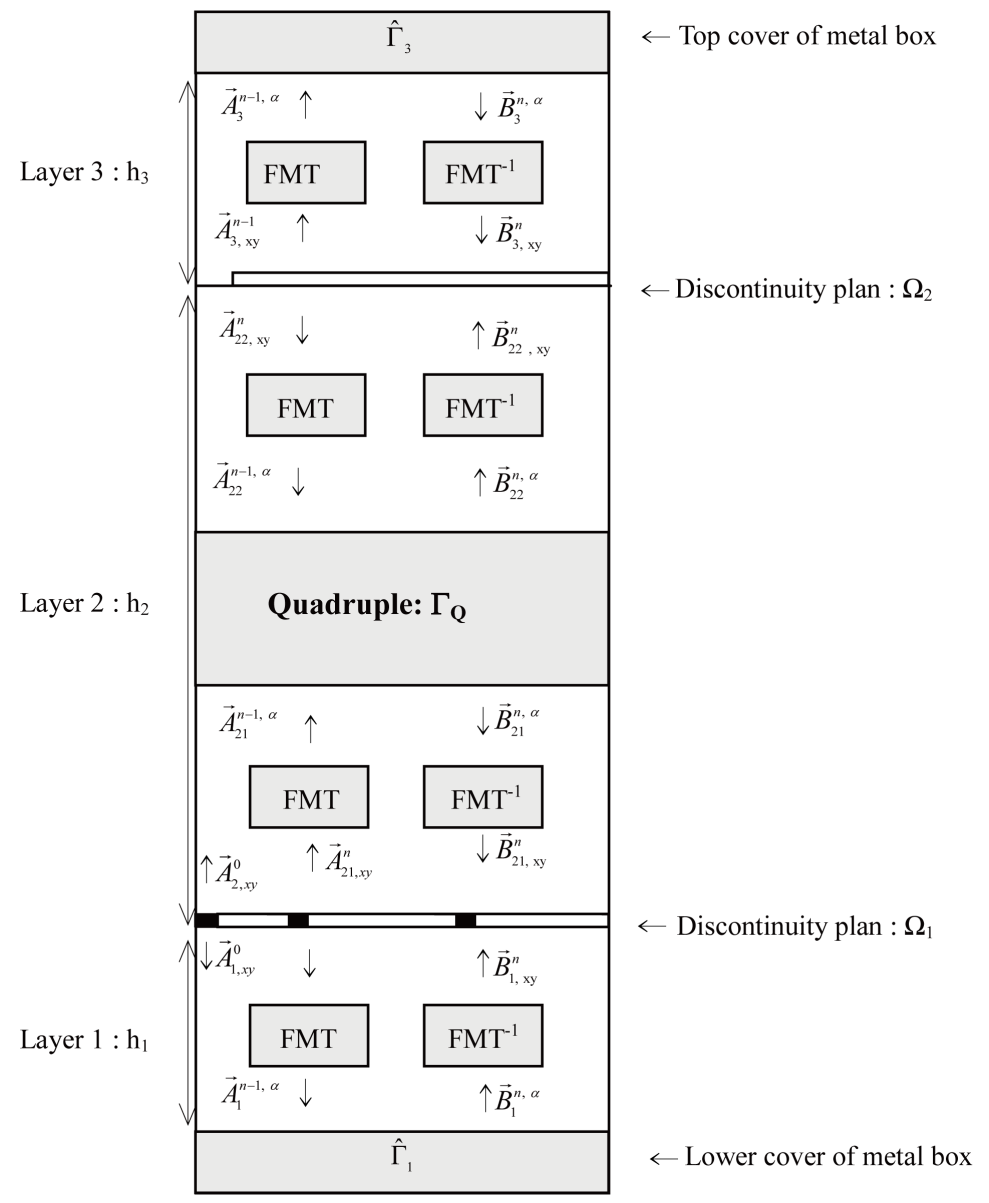

Figure 4. Synoptic diagram summarizing the iterative method for planar structures with three layers. 
placed at plans $\left(\Omega_{1}\right.$ and $\left.\Omega_{2}\right)$.

$\hat{\Gamma}_{k}$ : Operator reflection ensuring the link between the incident waves and the reflected waves. It is defined in the spectral domain. It contains information on the housing walls and the relative permittivity of the different mediums of the structure, $k \in$ \{medium 1 , medium 3$\}$.

$\hat{\Gamma}_{Q}$ : Diffraction Operator at each interface $\left(\Omega_{1}\right.$ and $\left.\Omega_{2}\right)$.

The evolution of iterations through the spectral domain to the space domain is done using the Fourier transform modal "FMT" which considerably reduces the calculation time. Modal Fourier transform requires the fragmentation of discontinuity planes $\left(\Omega_{1}\right.$ and $\left.\Omega_{2}\right)$ in pixels and this so that the electromagnetic behavior of the overall circuit will be summarized by writing the boundary conditions and continuity of the tangential fields on each pixel. The iterative process stops when it reaches the convergence of results.

The terms below link the incident waves " $\vec{A}_{1}, \vec{A}_{21}, \vec{A}_{22}$ and $\vec{A}_{3}$ ", to the reflected waves $\vec{B}_{1}, \vec{B}_{21}, \vec{B}_{22}$ and $\vec{B}_{3}$ " when they pass the space domain to the spectral domain:

$$
\begin{aligned}
& \left(\begin{array}{c}
A_{1} \\
A_{21}
\end{array}\right)=\hat{\Gamma}_{\Omega 1}\left(\begin{array}{c}
B_{1} \\
B_{21}
\end{array}\right)+\left(\begin{array}{l}
A_{1}^{0} \\
A_{2}^{0}
\end{array}\right) \\
& \left(\begin{array}{c}
B_{21} \\
B_{22}
\end{array}\right)=\hat{\Gamma}_{Q}\left(\begin{array}{c}
A_{21} \\
A_{22}
\end{array}\right) \\
& \left(\begin{array}{c}
A_{22} \\
A_{3}
\end{array}\right)=\hat{\Gamma}_{\Omega 2}\left(\begin{array}{c}
B_{22} \\
B_{3}
\end{array}\right) \\
& \left(\begin{array}{l}
\vec{J}_{21} \\
\vec{J}_{22}
\end{array}\right)=\left(\begin{array}{ll}
\hat{Y}_{11} & \hat{Y}_{12} \\
\hat{Y}_{21} & \hat{Y}_{22}
\end{array}\right)\left(\begin{array}{l}
\vec{E}_{21} \\
\vec{E}_{22}
\end{array}\right)
\end{aligned}
$$

The operators of diffraction $\hat{\Gamma}_{\Omega 1}$ and $\hat{\Gamma}_{\Omega 2}$ contains the images of circuit that being in the $\Omega_{1}$ and $\Omega_{2}$ plans. Figure 2(b) defines these two planes of discontinuities.

The flowchart in Figure 5 summarizes the evolution of the iterative method for a planar structure with three layers of different mediums.

- Diffraction Operator: $\hat{\Gamma}_{\Omega 1}$

For a source of bilateral excitation polarized in (oy), the overall diffraction operator is written from the diffraction operators in different regions of $\Omega_{1}$ plane (metal region, source region of excitement, dielectric region):

$$
\begin{aligned}
& {\left[\begin{array}{ll}
\hat{\Gamma}_{\Omega 1}\left(\begin{array}{l}
\mathrm{x} \\
\mathrm{y}
\end{array}\right)
\end{array}\right]=} \\
& {\left[\begin{array}{ll}
-\hat{H}_{m 1}-\left[\frac{Z_{01} Z_{02}+Z_{0}\left(Z_{01}-Z_{02}\right)}{Z_{01} Z_{02}+Z_{0}\left(Z_{01}+Z_{02}\right)}\right] \cdot \hat{H}_{S}+\frac{Z_{02}-Z_{01}}{Z_{01}+Z_{02}} \cdot \hat{H}_{i 1} & {\left[\frac{2 Z_{0} \sqrt{Z_{01} Z_{02}}}{Z_{01} Z_{02}+Z_{0}\left(Z_{01}+Z_{02}\right)}\right] \cdot \hat{H}_{S 1}+\frac{2 \sqrt{Z_{01} Z_{02}}}{Z_{01}+Z_{02}} \cdot \hat{H}_{i 1}} \\
\quad\left[\frac{2 Z_{0} \sqrt{Z_{01} Z_{02}}}{Z_{01} Z_{02}+Z_{0}\left(Z_{01}+Z_{02}\right)}\right] \cdot \hat{H}_{S 1}+\frac{2 \sqrt{Z_{01} Z_{02}}}{Z_{01}+Z_{02}} \cdot \hat{H}_{i 1} & -\hat{H}_{m 1}-\left[\frac{Z_{01} Z_{02}-Z_{0}\left(Z_{01}-Z_{02}\right)}{Z_{01} Z_{02}+Z_{0}\left(Z_{01}+Z_{02}\right)}\right] \cdot \hat{H}_{S 1}+\frac{Z_{01}-Z_{02}}{Z_{01}+Z_{02}} \cdot \hat{H}_{i 1}
\end{array}\right]}
\end{aligned}
$$

where: $H_{s 1}=1$ on the source and 0 elsewhere.

$H_{m 1}=1$ on the metal and 0 elsewhere.

$H_{i 1}=1$ on the dielectric and 0 elsewhere.

- Diffraction Operator: $\hat{\Gamma}_{\Omega 2}$

The overall diffraction operator is written from the diffraction operators in different regions of $\Omega_{2}$ plane (metal region, dielectric region):

$$
\left[\hat{\Gamma}_{\Omega 2\left(\begin{array}{l}
x \\
y
\end{array}\right)}\right]=\left[\begin{array}{cc}
-\hat{H}_{m 2}+\frac{Z_{02}-Z_{01}}{Z_{03}+Z_{02}} \cdot \hat{H}_{i 2} & \frac{2 \sqrt{Z_{03} Z_{02}}}{Z_{03}+Z_{02}} \cdot \hat{H}_{i 2} \\
\frac{2 \sqrt{Z_{03} Z_{02}}}{Z_{03}+Z_{02}} \cdot \hat{H}_{i 2} & -\hat{H}_{m 2}+\frac{Z_{03}-Z_{02}}{Z_{03}+Z_{02}} \cdot \hat{H}_{i 2}
\end{array}\right]
$$




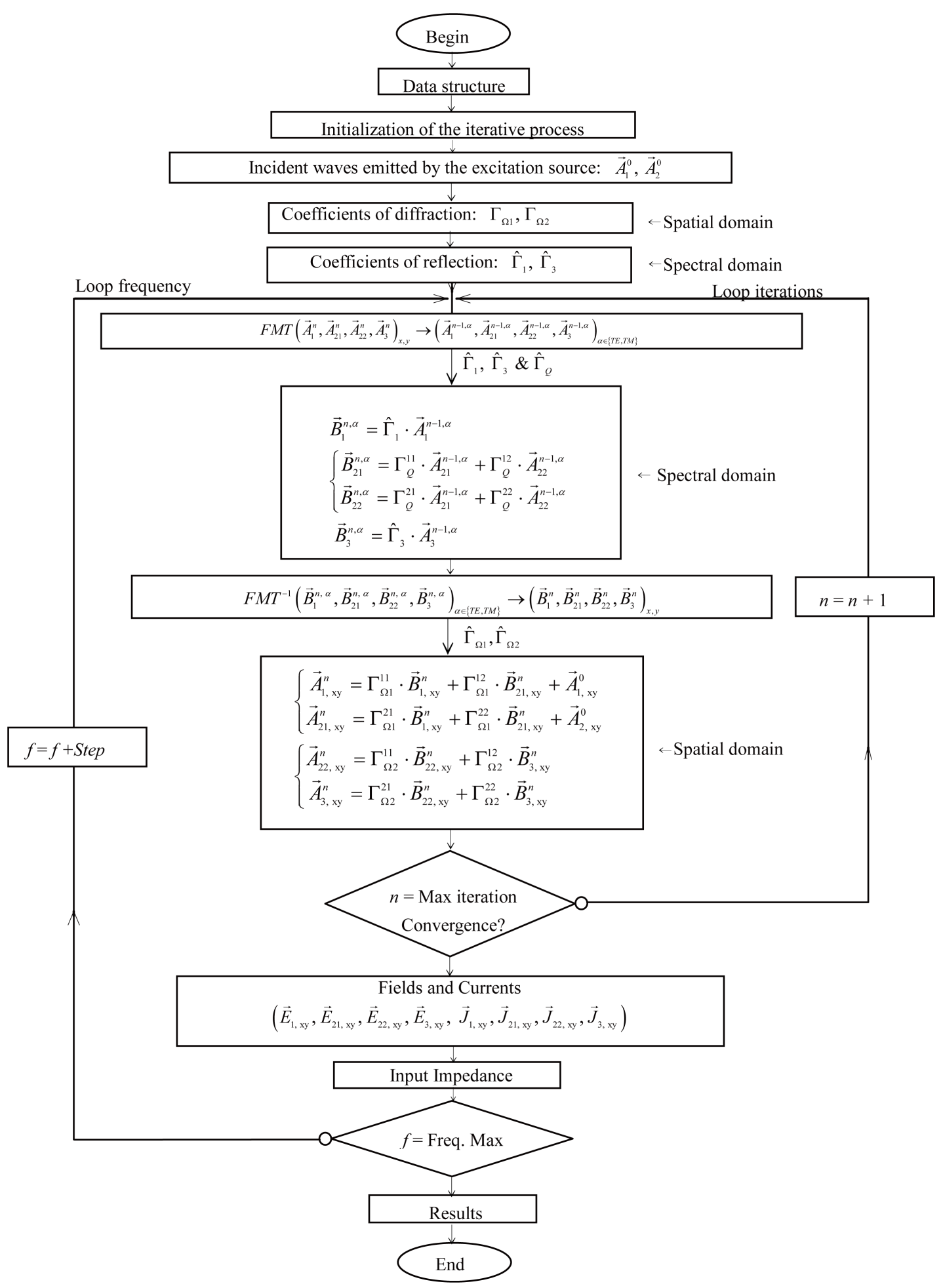

Figure 5. Flowchart summarizes the evolution of the iterative method for a planar structure with three layers of different mediums.

where: $H_{m 2}=1$ on the metal and 0 elsewhere.

$H_{i 2}=1$ on the dielectric and 0 elsewhere.

- Expression of the reflection operator: $\hat{\Gamma}_{k}$ 
It is defined in the spectral domain and contains information about the nature of the housing and the relative permittivity of the medium 1 and 3 of the structure. It is expressed by the following relationship:

$$
\hat{\Gamma}_{k}=\sum_{m, n, \alpha}\left|f_{m n}^{\alpha}\right\rangle \frac{1-Z_{0 k} Y_{m n}^{\alpha, k}}{1+Z_{0 k} Y_{m n}^{\alpha, k}}\left\langle f_{m n}^{\alpha}\right|
$$

$f_{m n}^{\alpha}$ : Basic functions. It depends on the nature of the box.

$Z_{0 k}=\frac{\eta}{\sqrt{\varepsilon_{r_{k}}}}:$ Impedance of the middle $k \in\{$ medium 1 , medium 2$\}$,

$\eta=120 \Pi:$ Vacuum impedance,

$Y_{m n}^{\alpha, k}:$ Mode admittance reduced to the level of $\Omega$ plan.

For a top cover (or lower) placed at a distance $h$ from $\Omega$ plan.

$$
Y_{m n}^{\alpha, k}=Y_{m n}^{\alpha}\left(\varepsilon_{r_{k}}\right) \cdot \operatorname{Coth}\left(\gamma_{m n}\left(\varepsilon_{r_{k}}\right) \cdot h\right)
$$

For an open circuit without top cover (or lower).

$$
Y_{m n}^{\alpha, k}=Y_{m n}^{\alpha}\left(\varepsilon_{r_{k}}\right)
$$

$Y_{m n}^{\alpha}\left(\varepsilon_{r_{k}}\right)$ : Mode admittance expressed by:

$$
\begin{aligned}
& T E: Y_{m n}^{T E}\left(\varepsilon_{r_{k}}\right)=\frac{\gamma_{m n}\left(\varepsilon_{r_{k}}\right)}{j \omega \mu_{0}} \\
& T M: Y_{m n}^{T M}\left(\varepsilon_{r_{k}}\right)=\frac{j \omega \varepsilon_{0} \varepsilon_{r_{k}}}{\gamma_{m n}\left(\varepsilon_{r_{k}}\right)}
\end{aligned}
$$

$\gamma_{m n}\left(\varepsilon_{r_{k}}\right)$ : Propagation constant

$$
\gamma_{m n}^{2}\left(\varepsilon_{r_{k}}\right)=\left(\frac{m \Pi}{a}\right)^{2}+\left(\frac{n \Pi}{b}\right)^{2}-k_{0}^{2} \varepsilon_{r_{k}}
$$

$k_{0}=\frac{\omega}{c}:$ Wave number in a vacuum.

$c=\frac{1}{\sqrt{\varepsilon_{0} \mu_{0}}}:$ Speed of light $\left(3 \times 10^{8} \mathrm{~m} / \mathrm{s}\right)$.

$m, n$ : Designating the index for modes $\in\{N\}$.

$\alpha$ : Mode indicator TE (Transverse Electric), TM (Transverse Magnetic).

$k$ : Medium considered $k \in\{1,2\}$.

$\varepsilon_{r_{k}}$ : Relative permittivity of the medium $k \in\{1,2\}$.

$\varepsilon_{0}$ : Vacuum permittivity $(\mathrm{F} / \mathrm{m})$.

$\mu_{0}$ : Magnetic vacuum permeability $(\mathrm{H} / \mathrm{m})$.

$\omega$ : Angular pulsation equal to pulsation $2 \cdot \Pi \cdot f(\mathrm{rd} / \mathrm{s})$.

\section{- Expression of the FMT}

The Fourier transform in cosine and sine is defined by:

$$
2 D-F F T_{\cos \sin }\left(\begin{array}{l}
E_{x}(i, j) \\
E_{y}(i, j)
\end{array}\right)=\left[\begin{array}{l}
\sum_{i=1}^{N_{01}} \sum_{j=1}^{N_{02}} E_{x}(i, j) \cdot \cos \left(\frac{m \Pi i}{N_{01}}\right) \cdot \sin \left(\frac{n \Pi j}{N_{02}}\right) \\
\sum_{i=1}^{N_{01}} \sum_{j=1}^{N_{02}} E_{y}(i, j) \cdot \sin \left(\frac{m \Pi i}{N_{01}}\right) \cdot \cos \left(\frac{n \Pi j}{N_{02}}\right)
\end{array}\right]
$$

The Fourier mode transform (FMT) is defined by:

$$
\left(\begin{array}{c}
e_{m n}^{T E} \\
e_{m n}^{T M}
\end{array}\right)=\hat{T} \cdot 2 D-F F T_{\cos \sin }\left(\begin{array}{l}
E_{x}(i, j) \\
E_{y}(i, j)
\end{array}\right)=F M T\left(\begin{array}{l}
E_{x}(i, j) \\
E_{y}(i, j)
\end{array}\right)
$$


$\hat{T}$ : Passing modal operator in the area expressed by:

$$
\begin{aligned}
& \hat{T}=K(m, n) \cdot\left[\begin{array}{cc}
\frac{n}{b} & -\frac{m}{a} \\
\frac{m}{a} & \frac{n}{b}
\end{array}\right] \\
& K(m, n)=\frac{1}{\sqrt{\left(\frac{m}{a}\right)^{2}+\left(\frac{n}{b}\right)^{2}}} \cdot \sqrt{\frac{2 \sigma_{m n}}{a b}} .
\end{aligned}
$$

\section{- The reflection operator of the Quadruple: $\hat{\Gamma}_{Q}$}

The reflection operator of the Quadruple is defined in layer 2 of the structure to be studied. It links the incident waves " $\vec{A}_{21}, \vec{A}_{22}$ " the reflected waves “ $\vec{B}_{21}, \vec{B}_{22}$ ” they pass the space domain to the spectral domain. The quadruple $Q$ ensures the passage of plan $\Omega_{1}$ to plan $\Omega_{2}$ and inversely (see Figure 2).

According to the diagram in Figure 6 we can write:

$$
\left(\begin{array}{l}
B_{21} \\
B_{22}
\end{array}\right)=\hat{\Gamma}_{Q}\left(\begin{array}{l}
A_{21} \\
A_{22}
\end{array}\right) .
$$

- Parameters $\hat{Y}_{i j}$ du quadruple Q:

$$
\begin{aligned}
& \left\{\begin{array}{l}
J_{21}=Y_{11} E_{21}+Y_{12} E_{22} \\
J_{22}=Y_{21} E_{21}+Y_{22} E_{22}
\end{array}\right. \\
& \left\{\begin{array}{l}
\overrightarrow{J_{0}}=\overrightarrow{J_{1}}+\overrightarrow{J_{21}} \\
\overrightarrow{J_{2}}=\overrightarrow{J_{22}}+\overrightarrow{J_{3}} \\
\overrightarrow{J_{1}}=Y_{h} \overrightarrow{E_{3}} \\
\overrightarrow{E_{1}}=\overrightarrow{E_{21}} \\
\overrightarrow{E_{22}}=\overrightarrow{E_{3}} .
\end{array}\right.
\end{aligned}
$$

The symmetry of the structure, allows us to write:

$$
\left\{\begin{array}{l}
\hat{Y}_{11}=\hat{Y}_{22} \\
\hat{Y}_{12}=\hat{Y}_{21}
\end{array} .\right.
$$

After some mathematically manipulation, it is possible to determine the matrix:

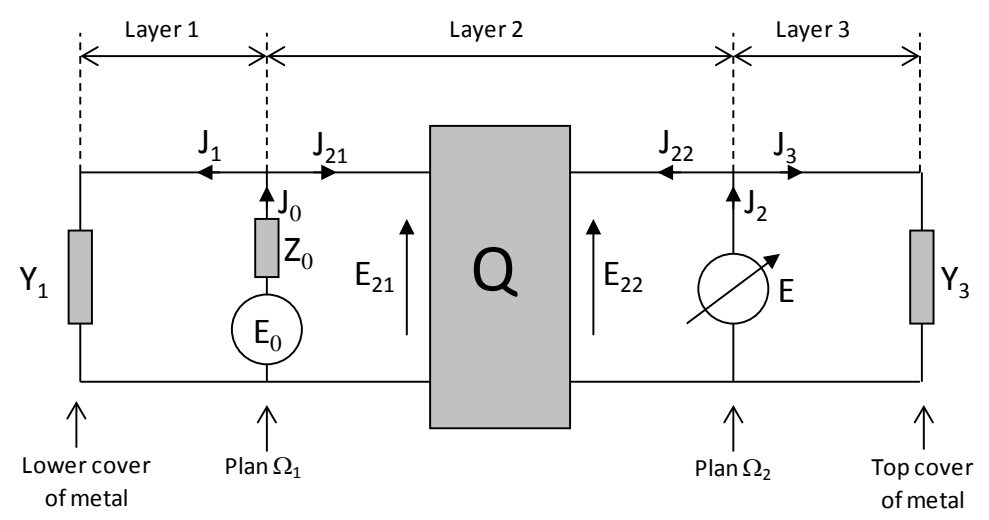

Figure 6. Equivalent electrical circuit of the planar structure including three layers of different medium. 


$$
\hat{\Gamma}_{Q}=\frac{1}{C}\left[\begin{array}{cc}
1-\left(Y_{11} Z_{02}\right)^{2}+\left(Y_{12} Z_{02}\right)^{2} & -2 Y_{12} Z_{02} \\
-2 Y_{12} Z_{02} & 1-\left(Y_{11} Z_{02}\right)^{2}+\left(Y_{12} Z_{02}\right)^{2}
\end{array}\right]
$$

with $C=\left(1+Y_{11} Z_{02}\right)^{2}-\left(Y_{12} Z_{02}\right)^{2}$

$$
\Gamma_{Q}=\sum_{m, n, \alpha}\left[\begin{array}{l|l}
\left|f_{m n}^{\alpha}\right\rangle \frac{1-\left(Y_{11} Z_{02}\right)^{2}+\left(Y_{12} Z_{02}\right)^{2}}{\left(1+Y_{11} Z_{02}\right)^{2}-\left(Y_{12} Z_{02}\right)^{2}}\left\langle f_{m n}^{\alpha}\right| & \left|f_{m n}^{\alpha}\right\rangle \frac{-2 Y_{12} Z_{02}}{\left(1+Y_{11} Z_{02}\right)^{2}-\left(Y_{12} Z_{02}\right)^{2}}\left\langle f_{m n}^{\alpha}\right| \\
\left|f_{m n}^{\alpha}\right\rangle \frac{-2 Y_{12} Z_{02}}{\left(1+Y_{11} Z_{02}\right)^{2}-\left(Y_{12} Z_{02}\right)^{2}}\left\langle f_{m n}^{\alpha}\right| & \left|f_{m n}^{\alpha}\right\rangle \frac{1-\left(Y_{11} Z_{02}\right)^{2}+\left(Y_{12} Z_{02}\right)^{2}}{\left(1+Y_{11} Z_{02}\right)^{2}-\left(Y_{12} Z_{02}\right)^{2}}\left\langle f_{m n}^{\alpha}\right|
\end{array}\right]
$$

$f_{m n}^{\alpha}$ : Bases function of the box modes.

$$
\left\{\begin{array}{l}
B_{21}^{\alpha}=\sum_{m, n, \alpha}\left|f_{m n}^{\alpha}\right\rangle\left(\frac{1-\left(Y_{11} Z_{02}\right)^{2}+\left(Y_{12} Z_{02}\right)^{2}}{\left(1+Y_{11} Z_{02}\right)^{2}-\left(Y_{12} Z_{02}\right)^{2}}\right)^{\alpha}\left\langle f_{m n}^{\alpha}\left|A_{21}^{\alpha}+\sum_{m, n, \alpha}\right| f_{m n}^{\alpha}\right\rangle\left(\frac{-2 Y_{12} Z_{02}}{\left(1+Y_{11} Z_{02}\right)^{2}-\left(Y_{12} Z_{02}\right)^{2}}\right)^{\alpha}\left\langle f_{m n}^{\alpha}\right| A_{22}^{\alpha} \\
B_{22}^{\alpha}=\sum_{m, n, \alpha}\left|f_{m n}^{\alpha}\right\rangle\left(\frac{-2 Y_{12} Z_{02}}{\left(1+Y_{11} Z_{02}\right)^{2}-\left(Y_{12} Z_{02}\right)^{2}}\right)^{\alpha}\left\langle f_{m n}^{\alpha}\left|A_{21}^{\alpha}+\sum_{m, n, \alpha}\right| f_{m n}^{\alpha}\right\rangle\left(\frac{1-\left(Y_{11} Z_{02}\right)^{2}+\left(Y_{12} Z_{02}\right)^{2}}{\left(1+Y_{11} Z_{02}\right)^{2}-\left(Y_{12} Z_{02}\right)^{2}}\right)^{\alpha}\left\langle f_{m n}^{\alpha}\right| A_{21}^{\alpha}
\end{array}\right.
$$

with $a \in\{T E, T M\}$.

\section{Convergence of the Method}

This study begins by checking the convergence of results based on iterations. This is to optimize the calculation time and improve the accuracy of the method. Figure 7 shows that the real part of the structure of the input impedance, converges from 3500 iterations and the imaginary part converges from 2000 iterations, for a frequency $f=1 \mathrm{GHz}$.

\section{Validation of the Method}

The simulation result (Figure 8) shows a comparison between a micro-strip line with a thick conductor and between a microstrip line with a conductor without thickness. These results show that our model simulation results find are identical with those calculated by Ansoft HFSS software, for a thick conductor. These results show the effectiveness of the correction made by our Model to the iterative method (FWCIP).

\section{Formulation of the Iterative Method for the Second Model}

\subsection{Studied Structure}

In this structure (Figure 9(a)) we have modeled the entire thick conductor. The upper and lower films of the conductor are modeled by two metal strips without thicknesses $(\delta=0)$, respectively placed in $\Omega_{1}$ and $\Omega_{2}$ plans. The interior of the thick film conductor and both of the two vertical sides are modeled by effective permittivity $\varepsilon_{\text {eff }}$ characterizing the layer 2 of the structure (Figure 9(b)). It is calculated from the complex relative permittivity of the thick conductor $\tilde{\varepsilon}^{\prime \prime}$ and the relative permittivity $\varepsilon_{r 2}=1$ of the air filling the remainder of the layer 2 of the study structure.

\section{- Calculus of the effective permittivity of the second layer:}

Figure 10 shows the equivalent model of the medium 2 located between the two planes $\Omega_{1}$ and $\Omega_{2}$ of Figure 9(b).

The permittivity of a metallic conductor is given by the following relationship:

$$
\tilde{\varepsilon}^{\prime \prime}=j \frac{\sigma}{2 \cdot \pi \cdot f}
$$

$\sigma$ : Conductivity $[\mathrm{S} / \mathrm{m}]$.

$f:$ Frequency $[\mathrm{Hz}]$. 


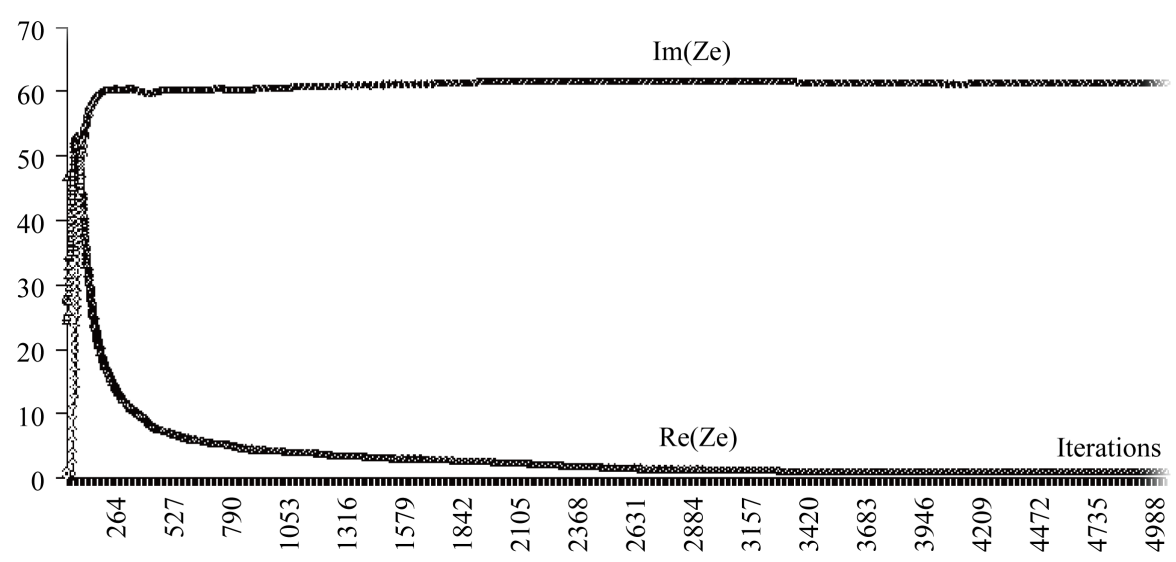

Figure 7. Convergence of Ze impedance based on iterations $(f=1 \mathrm{GHz})$.

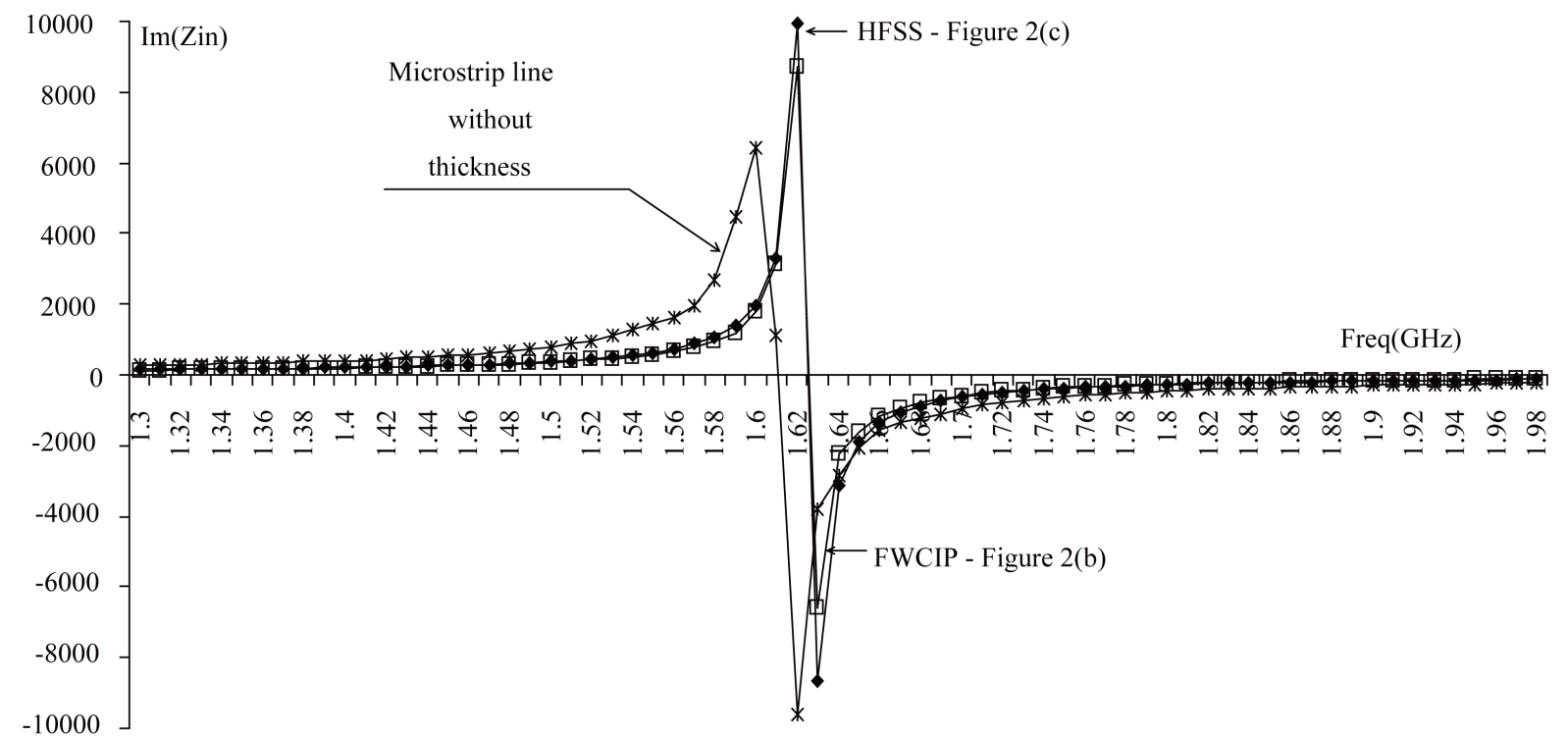

Figure 8. Comparison between a micro-strip line without thickness, micro-strip line with thickness (HFSS) and equivalent first model (FWCIP).

The effective permittivity " $\varepsilon_{\text {eff }}$ " is calculated from the relative permittivity of the medium 2 (second layer located between the planes $\Omega_{1}$ and $\Omega_{2}$ of the study structure (Figure 10). This medium consists of air $\varepsilon_{\mathrm{r} 2}=1$ and copper $\tilde{\varepsilon}^{\prime \prime}$ calculated by the relation (21).

The effective permittivity " $\varepsilon_{\text {eff }}$ " is calculated by the relation

$$
\varepsilon_{\text {eff }}=\left(\frac{\sum_{i} V_{i} \cdot \varepsilon_{r i}}{\sum_{i} V_{i}}\right)=\left(\frac{V_{1} \cdot \varepsilon_{r 2}+V_{2} \cdot \tilde{\varepsilon}^{\prime \prime}}{V_{1}+V_{2}}\right)=\left(\frac{S_{1} \cdot \varepsilon_{r 2}+S_{2} \cdot \tilde{\varepsilon}^{\prime \prime}}{S_{1}+S_{2}}\right)
$$

with:

$V_{1}$ : Volume of air which occupies the second layer.

$V_{2}$ : Volume of copper which occupies the second layer.

$S_{1}$ : Surface of air which occupies the plan $\Omega_{1}$.

$S_{2}$ : Surface of the copper occupies the plan $\Omega_{1}$.

$$
\varepsilon_{\text {eff }}=\left(\frac{S_{1}}{S_{1}+S_{2}}+j \frac{\sigma \cdot S_{2}}{\omega \cdot\left(S_{1}+S_{2}\right)}\right) .
$$




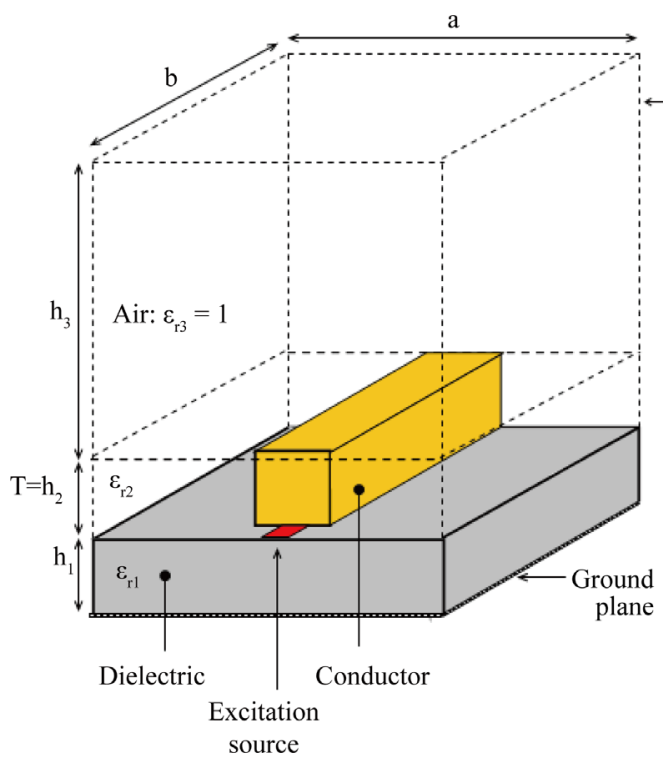

(a)

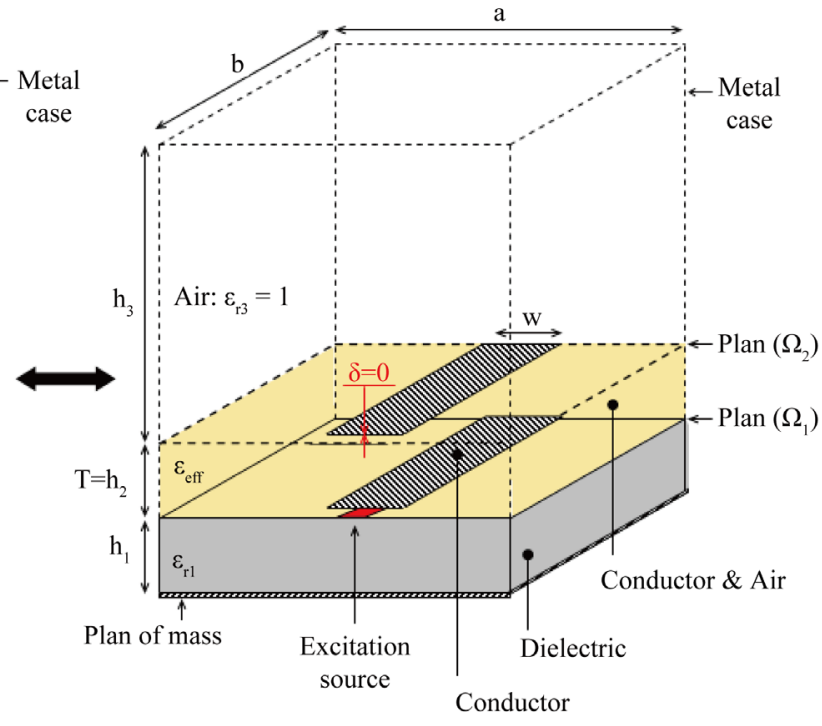

(b)

Figure 9. Studied structure and equivalent model.

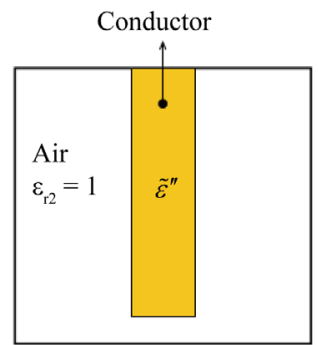

(a)

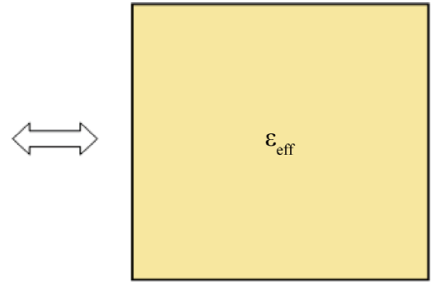

(b)

Figure 10. Discontinuity plan $\Omega 1$. (a): Prototype, (b) Proposed Model.

Therefore: $Z_{02 \_e f f}=\frac{\eta}{\sqrt{\varepsilon_{\text {eff }}}}=a+j b$.

$Z_{02 \_ \text {eff: }}$ Intrinsic impedance of the medium 2 (complex form).

The analysis of the two models is analogous, simply changer $\varepsilon_{r 2}=1$ by $\varepsilon_{e f f}$ who becomes in complex form, so we obtain:

At the planes $\Omega_{1}$ and $\Omega_{2}$ diffractions operators are given by the following matrix:

$\Gamma_{\Omega 1}=$

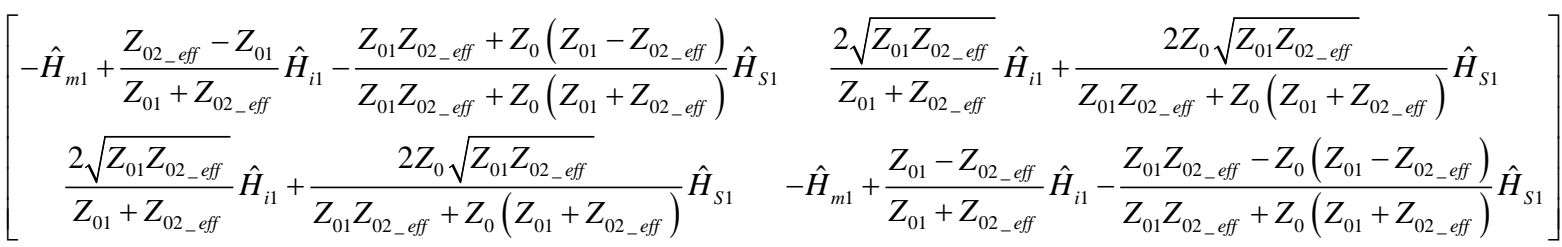

$$
\Gamma_{\Omega 2}=\left[\begin{array}{cc}
-\hat{H}_{m 2}+\frac{Z_{02 \_ \text {eff }}-Z_{03}}{Z_{03}+Z_{02 \_ \text {eff }}} \cdot \hat{H}_{i 2} & \frac{2 \sqrt{Z_{03} Z_{02 \_ \text {eff }}}}{Z_{03}+Z_{02 \_ \text {eff }}} \cdot \hat{H}_{i 2} \\
\frac{2 \sqrt{Z_{03} Z_{02 \_ \text {eff }}}}{Z_{03}+Z_{02 \_ \text {eff }}} \cdot \hat{H}_{i 2} & -\hat{H}_{m 2}+\frac{Z_{03}-Z_{02 \_ \text {eff }}}{Z_{03}+Z_{02 \_ \text {eff }}} \cdot \hat{H}_{i 2}
\end{array}\right] .
$$


And the diffraction operator $\hat{\Gamma}_{Q}$ at each interface $\left(\Omega_{1}\right.$ and $\Omega_{2)}$.is given by the following matrix:

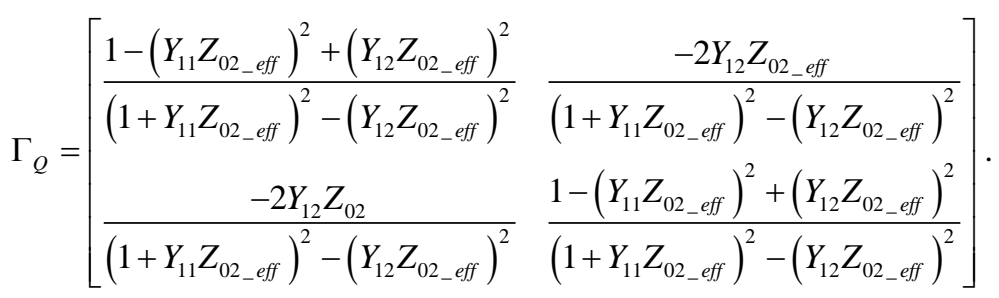

\subsection{Convergence of the Method}

This study begins by checking the convergence of results based on iterations. This is to optimize the calculation time and improve the accuracy of the method. Figure 11 shows that the real part of the structure of the input impedance, converges from 700 iterations and the imaginary part converges from 1500 iterations, for a frequency $f=1 \mathrm{GHz}$.

\subsection{Validation of the Analytical Method}

The simulation results (Figure 12) for a line with a thick conductor are in good agreement with those calculated by Ansoft HFSS software. For a micro-strip line with a driver without thickness the results obtained by the FWCIP method are shifted. This shows Indeed the use of exact model taking into account the thickness of the conductor seems necessary to improve the efficiency of the iterative method FWCIP.

These models tested showed adapting iterative method considered in the calculation of complex structures. Indeed, through the new formulation of the method, we showed a net correction of the resonance frequency observed in the case of structures without thickness. These models provide almost the same results (see Figure 13), requiring multilayer modeling structures.

We have shown the efficiency of the correction allocated to the iterative method by the two modals proposed for the modeling of the planar structures integrating the thick conductors. The results which were found and compared to those which were calculated by the HFSS software well demonstrate the improvement allocated to the iterative method.

\section{Conclusions}

This work allowed taking stock of two electromagnetic models with which we had characterized a planar structure including a flat, thick copper conductor. In fact, the first model based on the phenomenon of skin effect allowed us to model the latter with two metallic ribbons without thicknesses, placed one above the other with a distance $h_{2}$ equal to the thickness of the thick conductor. Both sides of the conductor have summers neglected

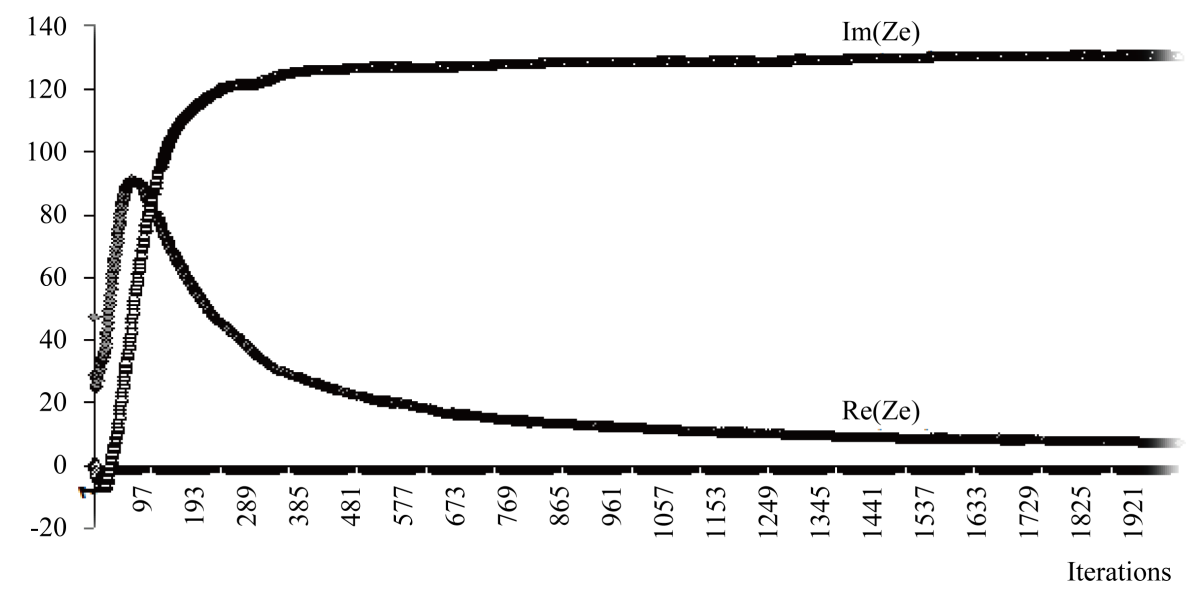

Figure 11. Convergence of Ze impedance based on iterations. 


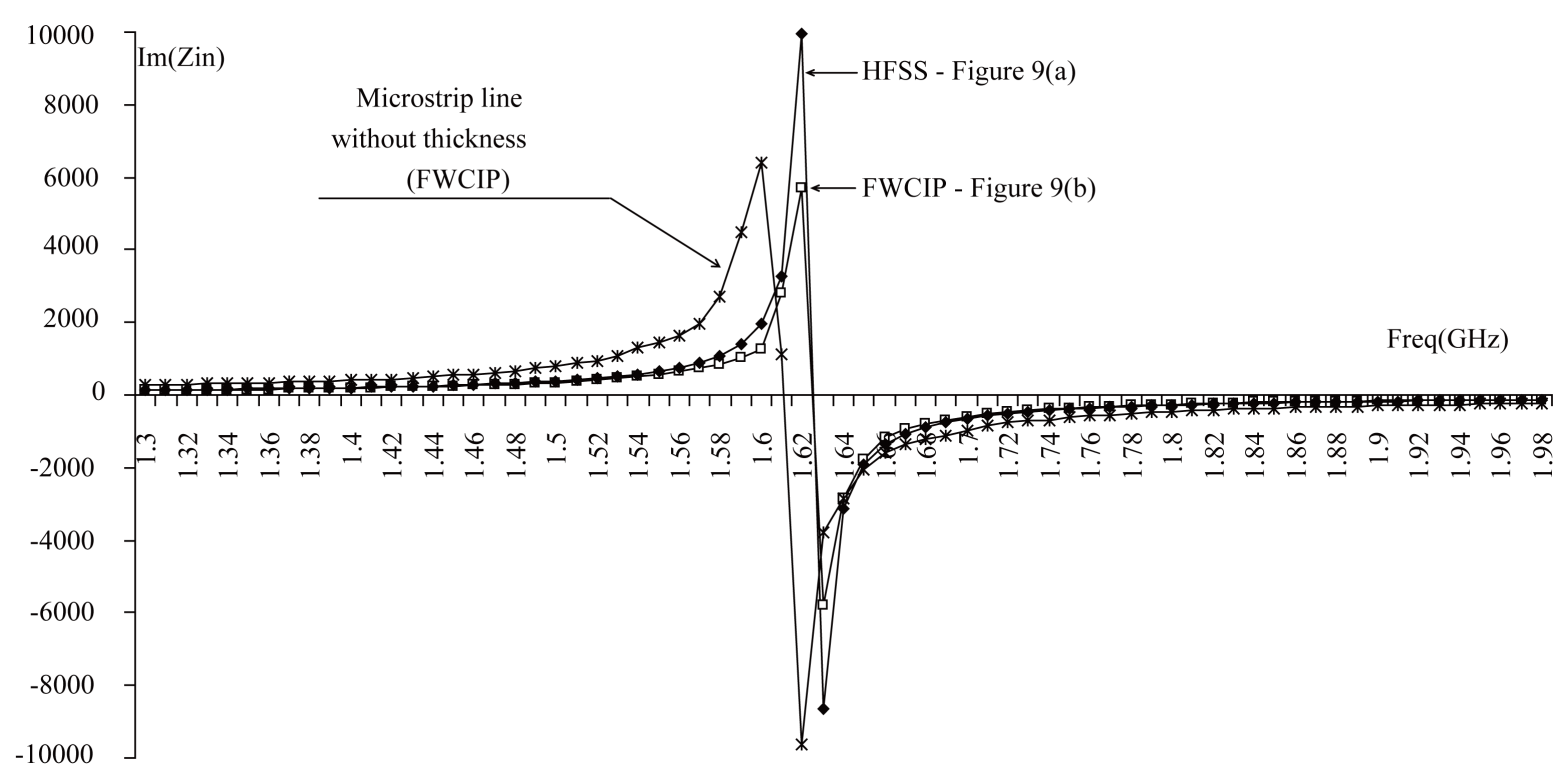

Figure 12. Structure study and simulation results.

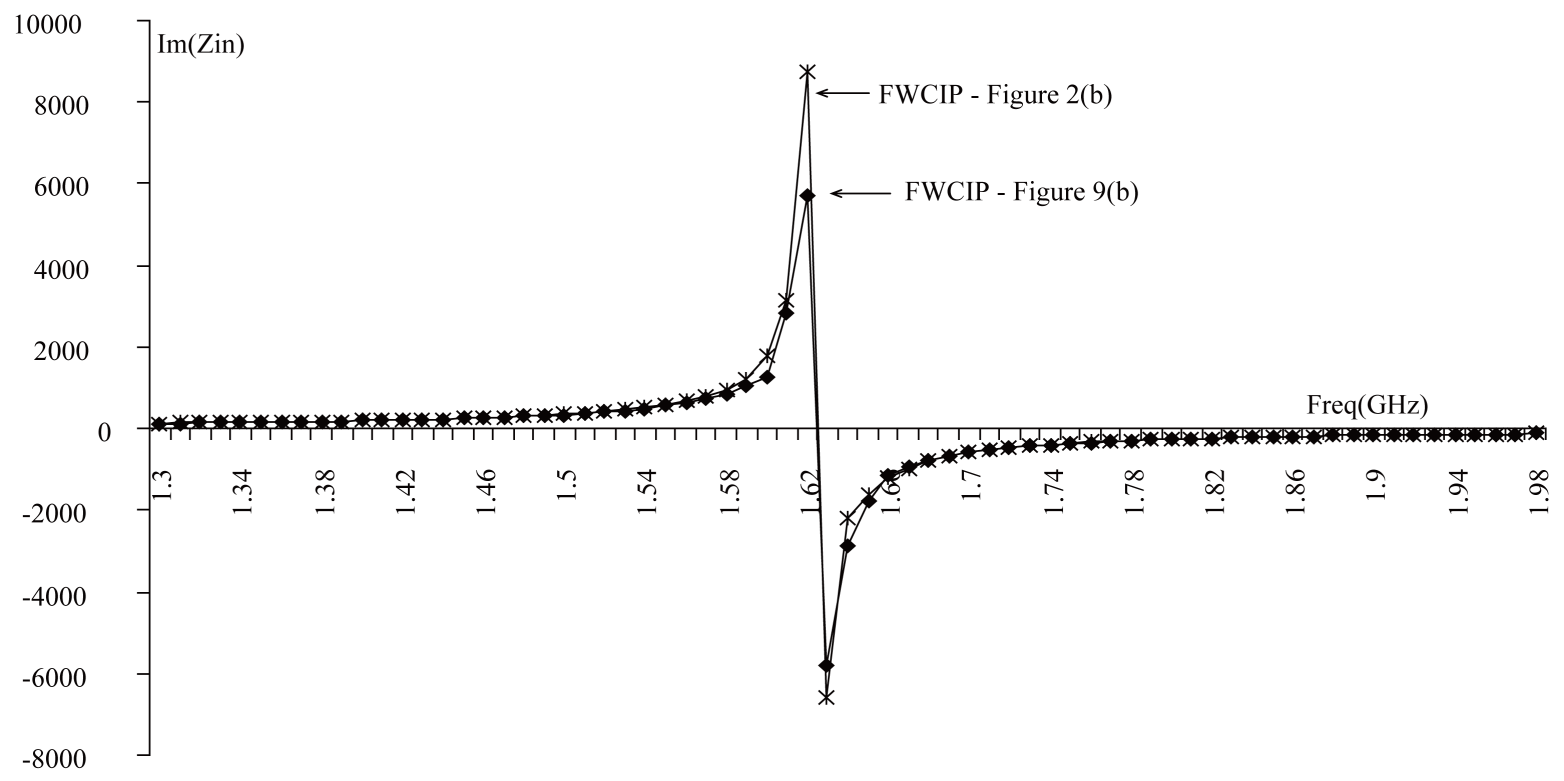

Figure 13. Comparison between the two proposed models.

since the width of the metal is strictly greater than its thickness. This is a simplifying assumption which has no effect on the results of the problem. This approach has been implemented and tested by the iterative method. Simulations results found were compared with those calculated by the software Ansoft HFFS, they were in good agreement, validating the method of analysis used. The second model is based on the calculation of the effective permittivity of the medium containing the thick conductor. This medium consists of a metallic region of complex relative permittivity, and the rest of this medium is filled with air $\varepsilon_{r 2}=1$. The effective permittivity $\varepsilon_{\text {eff }}$ calculated from these two relative permittivity $\varepsilon_{r 2}$ and $\tilde{\varepsilon}^{\prime \prime}$. Comparing the results of simulations of this new formulation of the iterative method with those calculated by the software Ansoft HFFS shows that they are in good agreement which validates the model. Both models tested in the iterative method have improved accuracy. These models generate almost the same results and require multilayer structures to be implemented.

The different cases of the structures studied in this article allowed to highlight the potential of iterative FWCIP and suggest that it would be an essential tool in the global modeling of planar structures with thick con- 
ductors.

\section{Acknowledgements}

This work has been supported by The SYSCOM laboratory, National Engineering School of Tunis Tunis El Manar University.

\section{References}

[1] Yeung, L.K. and Wu, K.-L. (2013) PEEC Modeling of Radiation Problems for Microstrip Structures. IEEE Transactions on Antennas and Propagation, 61, No. 7.

[2] Wang, X.-H., Zhang, H.L. and Wang, B.-Z. (2013) A Novel Ultra-Wideband Differential Filter Based on Microstrip Line Structures. IEEE Microwave and Wireless Components Letters, 23, No. 3.

[3] Serres, A., Serres, G.K.F., Fontgalland, G., Freire, R.C.S. and Baudrand, H. (2014) Analysis of Multilayer Amplifier Structure by an Efficient Iterative Technique. IEEE Transactions on Magnetics, 50, No. 2.

[4] Jan, J.-Y., Pan, C.-Y., Chang, F.-P., Wu, G.-J. and Huang, C.-Y. (2012) Realization of Compact and Broadband Performances Using the Microstrip-Line-Fed Slot Antenna. Proceedings of APMC 2012, Kaohsiung, Taiwan, 4-7 December 2012, 1379-1381.

[5] Kosslowski, S. (1988) The Application of the Point Matching Method to the Analysis of Microstrip Lines with Finite Metallization Thickness. IEEE Transactions on Microwave Theory and Techniques, 36, 1265-1271.

[6] Feng, N.N., Fang, D.D. and Huang, W.P. (1998) An Approximate Analysis of Microstrip Lines with Finite Metallization Thickness and Conductivity by Method of Lines. Proceedings of the International Conference on Microwave and Millimeter Wave Technology, 1998, Beijing, 1053-1056.

[7] Farina, M., et al. (2000) Spectral Domain Approach to 2D-Modelling of Open Planar Structures with Thick Lossy Conductors. IEE Proceedings-Microwaves, Antennas and Propagation, 147, 321. http://dx.doi.org/10.1049/ip-map:20000732

[8] Shih, C. (1989) Frequency-Dependent Characteristics of Open Microstrip Lines with Finite Strip Thickness. IEEE Transactions on Microwave Theory and Techniques, 37, 793-795.

[9] Henri, B., Sidima, W. and Damirnne, B. (2002) The Concept of Waves: Theory and Applications in Electronic Problems. Proceeding of the IX International Conference on Mathematical Methods in Electromagnetic Theory, Kiev, Ukraine, 10-13 September 2002, 100-104.

[10] Serres, A., Fontgalland, G., de Farias, J.E.P. and Baudrand, H. (2010) An Efficient Algorithm for Planar Circuits Design. IEEE Transactions on Magnetics, 46, No. 8.

[11] Kaddour, M., Mami, A., Gharsallah, A., Gharbi, A. and Baudrand, H. (2003) Analysis of Multilayer Microstrip Antennas by Using Iterative Method. Journal of Microwaves and Optoelectronics, 3, No. 1.

[12] Lamhene, Y., Tellache, M., Haraoubia, B. and Baudrand, H. (2009) Triangular Discretization for Analysis of Microstrip Mitred Bend, by an Iterative Method Using the Fast Modal Transform. International Journal of Computing, 8, 33-40. 